\title{
Influencia del viento y de la vegetación nativa en la distribución de la regeneración de Pseudotsuga menziesii en Patagonia, Argentina
}

\author{
Influence of wind and native vegetation on the distribution of Pseudotsuga menziesii \\ regeneration in Patagonia, Argentina
}

\author{
Laura Melania Acheritobehere ${ }^{\text {a*, }}$ Ivonne Alejandra Orellana a,b \\ *Autor de correspondencia: ${ }^{a}$ Universidad Nacional de la Patagonia San Juan Bosco, Facultad de Ciencias Naturales, \\ Departamento de Biología General, Ruta Nacional № 259 km 16,41, Esquel (9200) Chubut, Argentina, \\ tel.: (02945) 45-0807, lau_acheritobehere@hotmail.com \\ ${ }^{\mathrm{b}}$ Centro de Investigación y Extensión Forestal Andino Patagónico (CIEFAP), Esquel, Argentina.
}

\begin{abstract}
SUMMARY
Sapling distribution patterns of wind dispersed species tend to be influenced not only by wind direction and speed, but also by the presence of shrubs species that favor sapling recruitment. We analyzed the effect of wind direction and presence of understory species in Austrocedrus chilensis forest and shrublands, on Pseudotsuga menziesii sapling distribution. We selected three P. menziesii forestations; in each forestation, six transects were established in NE, E, SE, SW, W, NW orientations. Pseudotsuga menziesii seedling and sapling densities were evaluated in each transect. Understory species associated with $P$. menziesii saplings were also recorded. We found significant difference in P. menziesii sapling density between wind-favored versus non-favored orientations, being more abundant in wind-favored orientations. We also detected significant differences in P. menziesii saplings density associated with different understory species and herbaceous species or bare soil; the highest values were found in association with herbaceous species or with bare soil.
\end{abstract}

Key words: invasion, dispersal, Douglas fir, ciprés de la cordillera, saplings.

\section{RESUMEN}

Los patrones de distribución espacial de la regeneración de especies que se dispersan por anemocoria, suelen estar influenciados por la dirección del viento y también por la presencia de especies arbustivas del sotobosque que favorecen el establecimiento de la regeneración. Se analizó el efecto de la dirección del viento y de la vegetación asociada a bosques de Austrocedrus chilensis y matorrales mixtos, sobre la distribución de la regeneración de Pseudotsuga menziesii proveniente de plantaciones. Se seleccionaron tres plantaciones de P. menziesii; a partir de los bordes de cada una se establecieron seis transectas en las orientaciones NE, E, SE, SO, $\mathrm{O}, \mathrm{NO}$. En cada transecta se relevó la densidad de plántulas y renovales de $P$. menziesii y se registró las especies del sotobosque a las cuales estaba asociada la regeneración de $P$. menziesii. No se detectaron diferencias significativas en la densidad de plántulas en las diferentes orientaciones. En cambio, los renovales fueron más abundantes en las orientaciones favorecidas por el viento. Así mismo hubo una mayor frecuencia de renovales asociados a especies leñosas que a suelo desnudo o herbáceas. Estos resultados muestran cómo la dirección del viento y la facilitación de las especies leñosas afectan la dispersión de semillas y el establecimiento de los renovales y determinan los patrones de distribución de la regeneración de una especie arbórea.

Palabras clave: invasiones, dispersión, pino oregón, ciprés de la cordillera, renovales.

\section{INTRODUCCIÓN}

Las sustituciones de bosques nativos u otras comunidades naturales por forestaciones incrementan las posibilidades de ocurrencia de invasiones biológicas. Diferentes autores han documentado procesos de invasión de coníferas exóticas, a partir de plantaciones, en los bosques templados de Argentina (Simberloff et al. 2002, Sarasola et al. 2006, Orellana y Raffaele 2010) y Chile (Pauchard et al. 2010). Sin embargo, la relación entre la dispersión de las semillas con diferentes condiciones ambientales (e.g. dirección del viento), al igual que la relación entre el establecimiento de la regeneración y la estructura y composición de especies de las comunidades vegetales vecinas, son aspectos todavía poco estudiados.

En la Patagonia argentina, las forestaciones con $\mathrm{Pseu}$ dotsuga menziesii (Mirb.) Franco (pino oregón) se realizaron a partir de la década del cincuenta, en diferentes comunidades naturales como son los bosques de Nothofagus antarctica (G. Forster) Oersted (ñire), los bosques de Nothofagus pumilio (Poepp et Endl) Krasser (lenga), y especialmente en bosques de Austrocedrus chilensis 
(D. Don) Pic. Serm. et Bizzarri (ciprés de la cordillera) y matorrales mixtos (Orellana y Raffaele 2010). A partir de la década del ochenta, las plantaciones de $P$. menziesii comenzaron a realizarse cada vez más en áreas de ecotono bosque-estepa. Austrocedrus chilensis es una conífera endémica de los Bosques Templados de Sudamérica Austral, incluida en el Libro Rojo de IUCN en la categoría "casi amenazado" (Near Threatened - NT) (IUCN 2014). En los bosques de A. chilensis, bosques de Nothofagus spp. y matorrales mixtos hay antecedentes de invasiones causadas por P. menziesii y Pinus contorta Douglas (Simberloff et al. 2002, Sarasola et al. 2006, Orellana y Raffaele 2010). El conocimiento de factores tales como el inicio de la edad reproductiva, la producción de semillas, los mecanismos de dispersión de las especies, así como las interacciones de la regeneración con las especies del sotobosque, resultan claves para comprender la naturaleza de los procesos de invasión (Sarasola et al. 2006, Relva y Nuñez 2014). En Argentina, se ha observado que la reproducción de P. menziesii, se inicia muy tempranamente, alrededor de los 11 años (Orellana y Raffaele 2010). La polinización ocurre principalmente por anemocoria al igual que la dispersión (Hermann y Lavender 1990). En las especies que presentan dispersión por anemocoria, las semillas suelen quedar distribuidas en el suelo de forma heterogénea, aunque algunas características del clima como la velocidad y dirección del viento inciden considerable sobre su distribución, al igual que variables paisajísticas como la topografía y la vegetación acompañante (Rovere y Premoli 2005).

La dispersión de las semillas se considera la fase demográfica más importante para el movimiento de las plantas en el espacio, e influye fuertemente sobre los patrones de distribución espacial de las plántulas, especialmente, para aquellas especies que se reproducen sólo de manera sexual como son: A. chilensis, P. menziesii, P. contorta y Pinus ponderosa Dougl. ex Laws. (Rovere y Premoli 2005). Hay registros que indican que en los Bosques Templados de Sudamérica Austral la dispersión de semillas, así como los patrones de distribución de los renovales en torno a los parentales, suelen verse influenciados por la dirección del viento (Rovere y Premoli 2005, Sarasola et al. 2006). En general en Patagonia, los vientos del Oeste, suelen ser más intensos que los vientos en otras direcciones (Martínez 1982) y la dispersión de las semillas ocurre principalmente durante los meses de verano y otoño, con diferentes variantes según la fenología de las especies. Las semillas de $A$. chilensis y $P$. menziesii se dispersan principalmente durante los meses de otoño (Gobbi y Schlichter 1998).

La estructura de las comunidades vegetales y su dinámica está determinada en cierto grado por las interacciones que suceden entre las especies que la componen (Chalcoff et al. 2014). Estas interacciones se producen debido a que al crecer y desarrollarse una especie afecta los recursos disponibles y condiciones de crecimiento de otras especies, que coexisten espacial y temporalmente (Chalcoff $e t$ al. 2014). Estas interacciones se pueden dar entre distintas especies de árboles, entre árboles y arbustos (Kitzberger et al. 2000, Letourneau et al. 2004, Nuñez et al. 2009a), entre especies de arbustos (Raffaele y Veblen 1998), y entre árboles y gramíneas (Bava y Rechene 2004). Kitzberger et al. (2000) describieron como algunas plantas ejercen un efecto positivo sobre la regeneración de $A$. chilensis, las mismas durante el verano reducen la radiación incidente a nivel del suelo, la evapotranspiración, la temperatura del suelo y, probablemente, aumentan el contenido de materia orgánica y mejoran las condiciones de humedad del suelo. Por otro lado, las plantas nodriza de mayor tamaño (aproximadamente $30 \mathrm{~cm}$ de altura) producían un efecto negativo, suprimiendo el crecimiento en altura. Este comportamiento ha sido citado para otras coníferas en respuesta a bajas intensidades lumínicas. También se ha sugerido que las plantas nodrizas podrían facilitar la supervivencia de plántulas de $A$. chilensis en invierno, reduciendo el efecto del descalce producido por las heladas (Gobbi y Schlichter 1998). Se desconoce la relación de la regeneración de P. menziesii con las especies del sotobosque de los bosques de A. chilensis y los matorrales mixtos. En su lugar de origen $P$. menziesii precisa luz suave durante los primeros años de su establecimiento, y luego sol pleno. También se ha observado competencia de la regeneración con algunas especies propias del sotobosque y herbáceas. En su rango de distribución natural en Norteamérica, en general, la regeneración se establece de mejor manera con posterioridad a incendios, los cuales eliminan la competencia con la vegetación (Hermann y Lavender 1990).

Los objetivos de este trabajo son analizar la relación entre la distribución espacial de la regeneración de P. menziesii y la dirección del viento predominante en la región, al igual que la relación entre el establecimiento de la regeneración de $P$. menziesii con la vegetación de los bosques de $A$. chilensis y matorrales mixtos vecinos. Las hipótesis del trabajo son las siguientes: 1) existe mayor densidad de renovales de $P$. menziesii en las orientaciones favorecidas por la dirección del viento (E, NE y SE) que en las no favorecidas $(\mathrm{O}, \mathrm{NO}$ y $\mathrm{SO})$ y 2$)$ el sotobosque de los bosques de $A$. chilensis y matorrales mixtos favorece el establecimiento de la regeneración de $P$. menziesii.

\section{MÉTODOS}

Sistema en estudio. Pseudotsuga menziesii es una conífera introducida en Argentina, la especie es originaria de América del Norte, donde se extiende desde los $15^{\circ}$ de latitud Norte en México hasta los $55^{\circ}$ de latitud Norte en la Columbia Británica en Canadá. En Patagonia alcanza sus mayores crecimientos en laderas protegidas con exposiciones Este y Sudeste, las cuales se caracterizan por presentar suelos profundos y con gran retención de humedad (Davel 2008). Si bien la superficie plantada con $P$. menziesii es de aproximadamente 8.000 ha (Davel 2008), las plantaciones se encuentran ampliamente distribuidas desde los $40^{\circ}$ a los $43^{\circ}$ de latitud Sur y desde los $71^{\circ}$ a los $71^{\circ} 40^{\prime}$ de longitud 
Oeste, abarcando las provincias de Neuquén, Río Negro y Chubut. En el extremo Sur de esta zona, existen plantaciones de la especie a partir de la isohieta de $600 \mathrm{~mm}$ de precipitación anual y, en el extremo norte, a partir de los $1.000 \mathrm{~mm}$ anuales. En cuanto a la altitud se hallan aproximadamente entre los 300 y 1.100 m s.n.m. (Davel 2008).

Para el presente estudio se seleccionaron tres sitios de muestreo al noroeste de la Provincia del Chubut, Argentina. El sitio 1 (Baguilt) está ubicado en las cercanías de la Reserva Provincial Baguilt ( $43^{\circ} 12^{\prime} 12^{\prime \prime}$ S, $71^{\circ} 36^{\prime} 45^{\prime \prime}$ O), y los otros dos en el Parque Nacional Los Alerces: el sitio 2

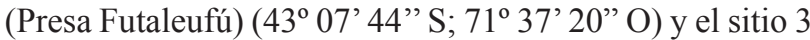

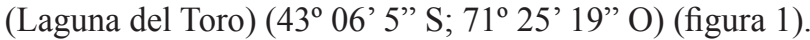
El clima es templado frío, con veranos cortos y secos e inviernos fríos y húmedos, con nevadas. Las precipitaciones en forma de lluvia, ocurren entre el otoño y el invierno, con una media de 800 a $1.200 \mathrm{~mm}$ anuales; mientras que las nevadas son comunes entre mayo y octubre. La temperatura media anual es de $8{ }^{\circ} \mathrm{C}$, y la velocidad promedio del viento en los últimos 10 años fue de $18,3 \mathrm{~km} \mathrm{~h}^{-1}$ con dirección predominante del Oeste (Martínez 1982). Los suelos de la región son de origen volcánico, y tienen

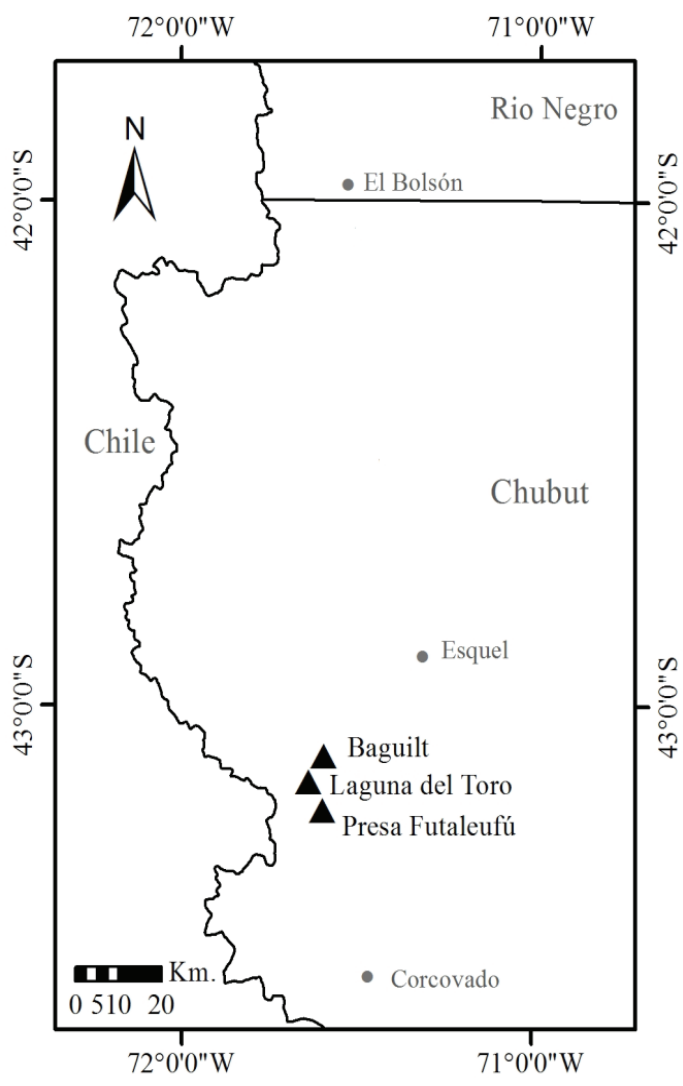

Figura 1. Mapa del área de estudio en la provincia de Chubut, donde se indican los tres sitios de estudio: Baguilt, Presa Futaleufú y Laguna del Toro.

Map of study area in Chubut Province, and sites: Baguilt, Presa Futaleufú and Laguna del Toro. una elevada capacidad de retención de humedad, alta capacidad de retención de fósforo y alto contenido de carbono orgánico. La vegetación está constituida por matorrales de $N$. antarctica, bosques de A. chilensis, y a mayor altitud, bosques de N. pumilio. También son muy frecuentes los matorrales mixtos de Lomatia hirsuta (Lam.) Diels, Schinus patagonicus (Phil.) I.M. Johbst. ex Cabrera, Maytenus boaria Molina, entre otras especies.

Método para el muestreo a campo. Se recorrió cada una de las plantaciones de $P$. menziesii seleccionadas y se midió su perímetro. El perímetro se dividió en seis distancias iguales, a partir del extremo con orientación Noreste. Se establecieron transectas partiendo desde el borde de la plantación en las siguientes orientaciones: $\mathrm{NE}, \mathrm{E}, \mathrm{SE}, \mathrm{SO}, \mathrm{O}$, $\mathrm{NO}$ (figura 2). Las orientaciones $\mathrm{N}$ y $\mathrm{S}$ fueron descartadas del muestreo, al no corresponder a los vientos dominantes.

Cada transecta, de $2 \mathrm{~m}$ de ancho por $120 \mathrm{~m}$ de largo, fue demarcada utilizando cinta métrica y una brújula modelo KB-14 360R, graduada de $0^{\circ}$ a $360^{\circ}$ para trazar el rumbo. En cada una de las transectas se establecieron parcelas de 2 metros de ancho x 10 metros de largo (unidad de muestreo). Resultando 12 unidades de muestreo instalados a los $10,20,30,40,50,60,70,80,90,100,110$ y 120 metros en cada una de las transectas. En cada transecta además se realizó una clasificación del tipo de vegetación o ambiente que rodeaba la plantación de P. menziesii: matorral, bosque de $A$. chilensis, quemado, camino. La edad de las

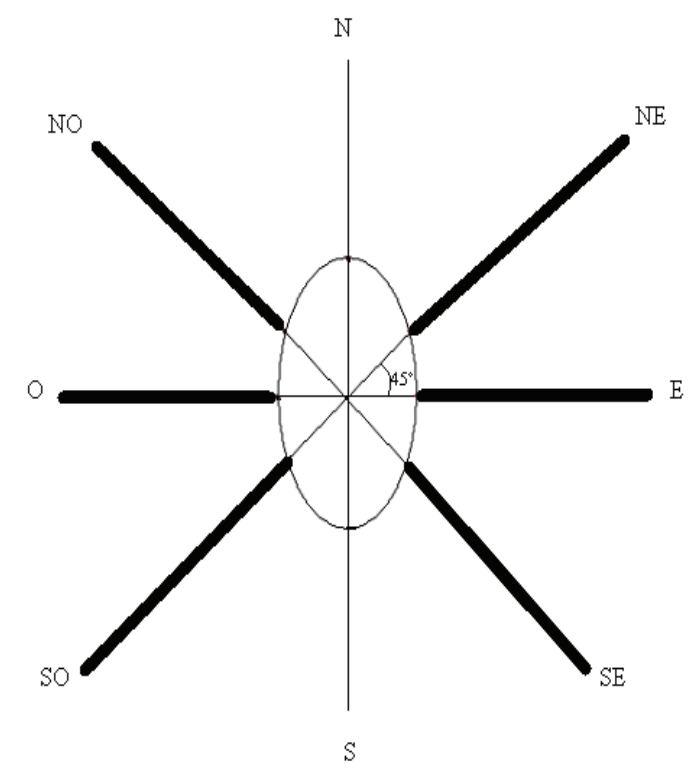

Figura 2. Diseño general de muestreo por sitio. El ovalo central representa una plantación, y las líneas negras representan las transectas en diferentes orientaciones. Cada transecta midió $120 \mathrm{~m}$ de largo x $2 \mathrm{~m}$ de ancho.

General sampling design by site. The central oval represents one forestation, black lines represent transects in all orientations studied. Each transect measured $120 \mathrm{~m}$ long $\mathrm{x} 2 \mathrm{~m}$ wide. 
plantaciones de $P$. menziesii se determinó en laboratorio, a partir de tarugos obtenidos con un barreno de incremento a 0,3 metros de altura. La diferencia en la edad debida a la altura fue corregida, agregando tres años (tiempo aproximado en que un renoval alcanza 0,3 metros de altura).

La edad de las plantaciones de $P$. menziesii estudiadas fue similar, con un promedio de $27 \pm 5$ años. En los tres sitios se registró establecimiento espontáneo de individuos reproductivos (i.e., con conos) de P. menziesii, a más de $100 \mathrm{~m}$ de las plantaciones (cuadro 1). Los ambientes que rodean la plantación de P. menziesii en el sitio 1, Baguilt, fueron: bosques de $\mathrm{A}$. chilensis y matorrales mixtos de $\mathrm{Fa}$ biana imbricata, Schinus patagonicus y L. hirsuta; también se observaron afloramientos rocosos con suelo poco desarrollado y la pendiente en este sitio es del $35 \%$. El sitio 2, Presa Futaleufú, se caracterizó por presentar sectores con pendientes del $50 \%$, en los que naturalmente se desarrollan: bosques de $A$. chilensis y matorrales mixtos, $\mathrm{o}$ existen roquedales con muy poco desarrollo de vegetación. Además, se observaron diferentes tipos de disturbios, como caminos y restos de poda de $P$. menziesii en los sectores E, SE y O. En el sitio 3, Laguna del Toro, la plantación se encontraba rodeada de matorral de $N$. antarctica, bosque de A. chilensis y matorral de F. imbricata. Esta plantación se encuentra situada en pendientes del orden del $30 \%$.

Densidad de regeneración de P. menziesii. Se establecieron dos categorías de tamaño de la regeneración: plántulas y renovales. La categoría plántulas incluyo a los individuos menores a 10 centímetros de altura, y sin ramificaciones laterales. La categoría renovales incluyo a los individuos mayores a 10 centímetros de altura y con ramificaciones laterales. Se evaluó la densidad de plántulas y renovales en cada una de las unidades de muestreo.

Asociación de los renovales de P. menziesii con especie del sotobosque. Se identificaron las especies arbóreas, arbustivas y enredaderas del sotobosque, a las que se encontraban asociados los renovales de P. menziesii. Se consi- deró que los renovales de $P$. menziesii estaban asociados a otras especies cuando la distancia entre ellos era menor a un radio de copa de la especie asociada. En ocasiones los renovales se establecían en suelo desnudo o con herbáceas. Cada especie arbórea o arbustiva a la que se asociaban los renovales de $P$. menziesii fue colectada, herborizada y, posteriormente, identificada en el laboratorio. Debido a que las plántulas suelen ser efímeras, no fueron contempladas en este análisis.

Comparación de la densidad de plántulas y renovales de $\mathrm{P}$. menziesii, en las orientaciones favorecidas y no favorecidas por la dirección del viento. Se comparó la densidad de individuos en las orientaciones favorecidas por la dirección del viento (E, NE y SE) versus las no favorecidas $(\mathrm{O}, \mathrm{NO}$ y SO) mediante una prueba de comparación de pendientes. Para obtener los modelos lineales a comparar se realizaron regresiones lineales simples. Las variables dependientes fueron: 1) la densidad de plántulas de P. menziesii en las orientaciones Oeste (que incluye las orientaciones $\mathrm{O}, \mathrm{NO}$ y SO) y 2) la densidad de plántulas de $P$. menziesii en las orientaciones Este (que incluye las orientaciones E, NE y $\mathrm{SE})$. La variable independiente fue la distancia a la línea de borde de la plantación. Se repitió el mismo análisis para la densidad de renovales.

Análisis de la relación de los renovales de P. menziesii con las especies del sotobosque. Se comparó la densidad de renovales de P. menziesii mediante una Prueba de Friedman donde los niveles fueron: 1) especies leñosas y 2) suelo desnudo o herbáceas. También se comparó la densidad de renovales de $P$. menziesii asociados a distintas especies leñosas, para aquellas especies con mayores frecuencias de asociaciones, mediante otra Prueba de Friedman. La variable dependiente fue la densidad de renovales de P. menziesii y los niveles cada una de las siguientes especies: $A$. chilensis, F. imbricata, S. patagonicus, L. hirsuta, M. boaria y $N$. antarctica. Se utilizó el programa Infostat (Di Rienzo et al. 2008).

Cuadro 1. Características de las plantaciones de P. menziesii (perímetro, superficie, edad) y los ambientes naturales (vegetación aledaña, disturbios) en los tres sitios de muestreo.

Forestations and sampling site characteristics.

\begin{tabular}{lccccc}
\hline \multicolumn{1}{c}{ Sitio } & $\begin{array}{c}\text { Perímetro } \\
(\mathrm{m})\end{array}$ & $\begin{array}{c}\text { Superficie } \\
(\mathrm{ha})\end{array}$ & $\begin{array}{c}\text { Edad } \\
(\mathrm{años})\end{array}$ & Tipo de vegetación aledaña & Otros disturbios \\
\hline 1) Baguilt & 2.153 & 3,7 & 19 & $\begin{array}{c}\text { Bosque de } \text { A. chilensis } \\
\text { Matorral mixto }\end{array}$ & $\begin{array}{c}\text { Ganadería } \\
\text { Senderos turísticos } \\
\text { 2) Presa Futaleufú }\end{array}$ \\
$\begin{array}{llll}\text { Caminos } \\
\text { 3) Laguna del Toro }\end{array}$ & 318 & 0,2 & 26 & $\begin{array}{c}\text { Bosque de } \text { A. chilensis } \\
\text { Matorral mixto }\end{array}$ & $\begin{array}{c}\text { Tendidos de luz } \\
\text { Restos de poda }\end{array}$ \\
\end{tabular}




\section{RESULTADOS}

Comparación de la densidad de plántulas y renovales de P. menziesii, en las orientaciones favorecidas versus no favorecidas por la dirección del viento en función de la distancia a la plantación. El modelo [1] describe la densidad promedio de plántulas de $P$. menziesii para las orientaciones E, NE y SE (favorecidas por la dirección del viento), en función de la distancia a la línea de borde de la plantación (figuras 3A y 4A). Mientras que el modelo [2] describe la densidad promedio de plántulas de $P$. menziesii para las orientaciones $\mathrm{O}, \mathrm{NO}$ y $\mathrm{SO}$ (no favorecida por la dirección del viento), en función de la distancia a la línea de borde de la plantación (0 metros) (figuras 3A y 4A).

$$
\begin{aligned}
& \mathrm{y}=327.898-3.019(\mathrm{x}) \mathrm{R}^{2}=0,652 \quad P=0,008 \\
& \mathrm{y}=393.833-4.031(\mathrm{x}) \quad \mathrm{R}^{2}=0,608 \quad P=0,003
\end{aligned}
$$

Donde,

$\mathrm{y}=$ densidad, medida en individuos $\mathrm{ha}^{-1}$

$\mathrm{x}=$ distancia, medida en metros $(\mathrm{m})$

La densidad de plántulas de $P$. menziesii justo en el borde de las plantaciones es alta, tanto en las orientaciones no favorecidas, como en las orientaciones favorecidas por la dirección del viento (ver ordenadas de los modelos [1] y [2]). En ambos modelos, conforme aumenta la distancia a la plantación la densidad de plántulas disminuye (pendiente negativa). Cuando se compararon las pendientes de los modelos de regresión para la densidad de plántulas de
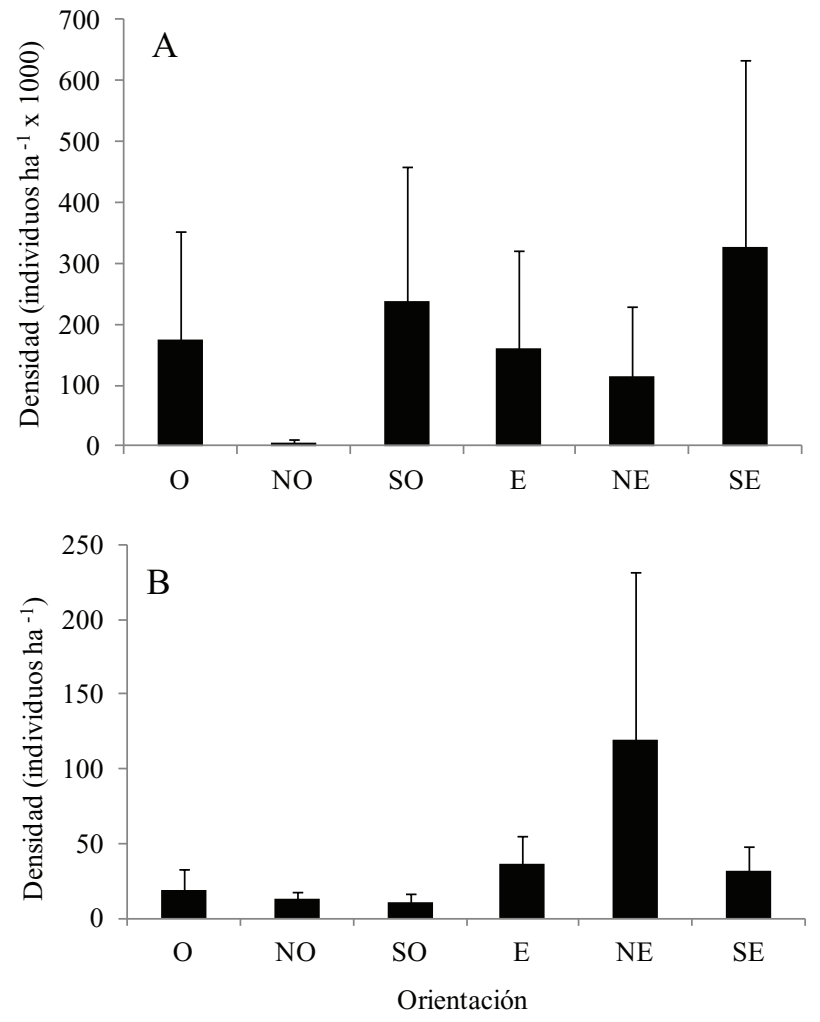

Figura 3. Densidad de plántulas A) y de renovales B) de P. menziesii $( \pm \mathrm{ES})$ en diferentes orientaciones, $\mathrm{n}=3$ sitios.

Pseudotsuga menziesii seedling density $( \pm \mathrm{ES})$ in different orientations, $\mathrm{n}=3$ sites. Differences are not significant.
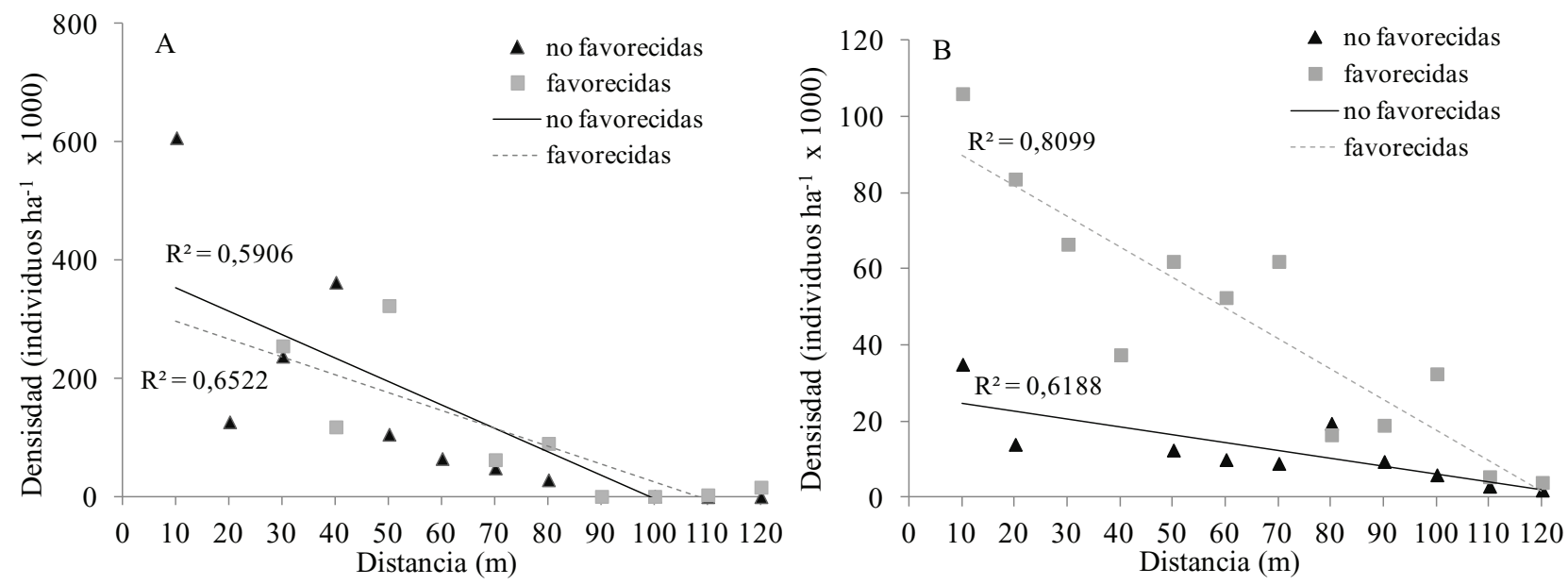

Figura 4. Modelos de regresión lineal simple para la densidad de plántulas A) y de renovales B) de P. menziesii (individuos ha-1 ), en función de la distancia, en las orientaciones favorecidas y no favorecidas por la dirección del viento. La diferencia de las pendientes no fue significativa para plántulas y significativas $\left(\mathrm{t}_{20}=4,4 ; P=0,0002\right)$ para renovales.

Simple linear regression models for $P$. menziesii (individuals ha ${ }^{-1}$ ) seedling A) and sapling B) density in function of distance, in wind direction favored and non-favored orientations. The difference of slopes was not significant for seedlings and significant $\left(\mathrm{t}_{20}=4.4 ; P=0.0002\right)$ for saplings. 
$P$. menziesii en las orientaciones favorecidas y no favorecidas, no se observaron diferencias significativas $\left(\mathrm{t}_{20}=0,8\right.$; $P=0,4)$.

El modelo [3] describe la densidad promedio de renovales de $P$. menziesii en las orientaciones E, NE y SE (favorecidas por la dirección del viento), en función de la distancia a la línea de borde de la plantación (figuras 3B y 4B). Mientras que el modelo [4] describe la densidad promedio de renovales de $P$. menziesii en las orientaciones $\mathrm{O}, \mathrm{NO}$ y SO (no favorecidas por la dirección del viento) en función de la distancia a la línea de borde de la plantación (0 metros) (figuras 3B y 4B).

$$
\begin{array}{lll}
\mathrm{y}=97.506-798(\mathrm{x}) & \mathrm{R}^{2}=0,81 & P<0,001 \\
\mathrm{y}=26.646-205(\mathrm{x}) & \mathrm{R}^{2}=0,62 & P=0,007
\end{array}
$$

Donde,

$\mathrm{y}=$ densidad, medida en individuos $\mathrm{ha}^{-1}$

$\mathrm{x}=$ distancia, medida en metros $(\mathrm{m})$.
La densidad de renovales de $P$. menziesii justo en el borde de las plantaciones es alrededor de tres veces mayor en las orientaciones favorecidas por la dirección del viento (ver ordenada de los modelos [3] y [4]). En ambos modelos, al igual que en los de la sección anterior, conforme aumenta la distancia a la plantación, la densidad de renovales disminuye (pendiente negativa). Las pendientes de los modelos de regresión para la densidad de renovales de $P$. menziesii en las orientaciones favorecidas y no favorecidas registraron diferencias significativas $\left(\mathrm{t}_{20}=4,4\right.$; $P=0,0002)$. La densidad de renovales es mayor en las orientaciones favorecidas.

Relación entre la densidad de renovales de P. menziesii y las especies del sotobosque. De un total de 2.096 renovales analizados, el 65,6 \% se encontró asociado a 22 especies leñosas y el 34,4 \% a suelo desnudo o herbáceas. La frecuencia de renovales asociados a cada especie leñosa, suelo desnudo o herbáceas se presenta en el cuadro 2.

Cuando se comparó la densidad de renovales de P. menziesii asociados a especies leñosas o a suelo des-

Cuadro 2. Frecuencia de renovales de $P$. menziesii asociados a diferentes especies leñosas del sotobosque o a suelo desnudo o herbáceas.

\begin{tabular}{|c|c|c|}
\hline Especie o grupo funcional & Hábito & Frecuencias $\left(\mathrm{n}^{\circ}\right.$ individuos $\left.\mathrm{ha}^{-1}\right)$ \\
\hline Austrocedrus chilensis (D. Don) Pic. Serm. et Bizzarri & Árbol & 359 \\
\hline Fabiana imbricata Ruiz et Pav. & Arbusto & 270 \\
\hline Schinus patagonicus (Phil.) I.M. Johnst. ex Cabrera & Árbol & 239 \\
\hline Lomatia hirsuta (Lam.) Diels & Árbol & 125 \\
\hline Maytenus boaria Molina & Árbol & 122 \\
\hline Nothofagus antarctica (G. Forst.) Oerst. & Árbol & 71 \\
\hline Gaultheria poeppigii DC. & Arbusto & 28 \\
\hline Colletia hystrix Clos & Arbusto & 26 \\
\hline Mutisia spinosa Ruiz et Pav. & Enredadera & 25 \\
\hline Mutisia decurrens Cav. & Enredadera & 24 \\
\hline Cytisus scoparius (L.) Link & Arbusto & 15 \\
\hline Rosa rubiginosa $\mathrm{L}$. & Arbusto & 15 \\
\hline Discaria chacaye (G. Don) Tortosa & Arbusto & 13 \\
\hline Maytenus chubutensis (Speg.) Lourteig, O`Donell et Sleumer & Árbol & 11 \\
\hline Aristotela chilensis (Molina) Stuntz & Arbusto & 6 \\
\hline Baccharis obovata Hook. et Arn. & Arbusto & 5 \\
\hline Embothrium coccineum J.R. Forst. et G. Forst. & Arbusto & 5 \\
\hline Berberis microphylla G. Forst. & Arbusto & 4 \\
\hline Discaria americana Gill. et Hook. & Arbusto & 4 \\
\hline Discaria articulata (Phil) Miers & Arbusto & 4 \\
\hline Ribes magellanicum Poir. & Arbusto & 2 \\
\hline Suelo desnudo o herbáceas & - & 722 \\
\hline
\end{tabular}
Pseudotsuga menziesii sapling frequency associated with different understory woody species, bare soil or herbaceous species. 
nudo o herbáceas, se detectaron diferencias significativas $\left(\mathrm{T}^{2}{ }_{1}=1 \mathrm{E} 30, P<0,0001\right)$. La mayor densidad de renovales de $P$. menziesii estuvo asociada a especies leñosas. En tanto, no hubo diferencias significativas en la frecuencia de renovales de $P$. menziesii asociados a las especies: $A$. chilensis, F. imbricata, S. patagonicus, L. hirsuta, M. boaria y $N$. antarctica $\left(\mathrm{T}^{2}{ }_{5}=2,80, P=0,0779\right.$; figura 5).

\section{DISCUSIÓN Y CONCLUSIONES}

La presencia de renovales reproductivamente maduros a más de 100 metros de las plantaciones, confirma la ocurrencia de un proceso de invasión de $P$. menziesii, de acuerdo a la definición dada por Richardson et al. (2000) para invasiones de especies arbóreas. Estos resultados, coinciden con los de Sarasola et al. (2006) en el Oeste de Neuquén, al igual que con los de Orellana y Raffaele (2010) en el Oeste de Río Negro y Chubut, quienes documentaron invasiones de $P$. menziesii en bosques de A. chilensis, matorrales mixtos y estepa.

Los renovales y plántulas de $P$. menziesii se distribuyen, en general, en toda el área de borde que rodea las plantaciones, hasta los 120 metros analizados en este estudio, tanto en las orientaciones favorecidas como en las no favorecidas por la dirección del viento. La regeneración se establece en diferentes ambientes, ya sean: vegetación natural circundante, suelo desnudo, o áreas muy modificadas (caminos, sendas). Varios autores han remarcado la importancia de las áreas de borde como facilitadores de las invasiones, debido a que suelen ser ambientes con mayor nivel de disturbio y ofrecen excelentes oportunidades para el establecimiento (Richardson et al. 2000, Orellana y Raffaele 2010).

Los ambientes de borde de las plantaciones, además ser ambientes con mayor nivel de disturbio, también reciben un mayor aporte de micorrizas y propágulos de $P$. menziesii, que contribuyen al proceso de invasión. Tanto las plántulas como los renovales, presentan un patrón de distribución en el cual hay altas densidades en torno a la plantación y luego la densidad disminuye a medida que aumenta la distancia a las plantaciones (i.e. las fuentes de propágulos). Estos resultados confirman la hipótesis de Williams et al. (2006), quienes proponen que la mayor parte de las semillas se dispersan a cortas distancias del árbol madre, entre los 10 y $140 \mathrm{~m}$ a partir de los árboles semilleros, fenómeno conocido como dispersión dentro del vecindario, o a corta distancia. Así mismo, recientemente se dilucidó la naturaleza de la relación entre las micorrizas y los procesos de invasión de $P$. menziesii en la región, estos estudios demuestran que el establecimiento de la regeneración, depende fuertemente, de la distancia alcanzada por el inóculo micorricico previamente al establecimiento de los renovales (Nuñez et al. 2009b, Salgado Salomón et al. 2013). Las plantaciones constituyen ambientes fuente de: 1) el inóculo micorrícico, que se establece tempranamente y 2) de los propágulos (semillas) que se establecen con posterioridad.

Cuando se analizaron los patrones de densidad de plántulas de $P$. menziesii en áreas favorecidas por la dirección del viento (NE, E y SE), versus las no favorecidas (NO, O, $\mathrm{SO})$, no se encontraron diferencias significativas. La ausencia de un efecto de la dirección del viento sobre la distribución de las plántulas de $P$. menziesii, puede deberse a la ocurrencia de un evento puntual, de escasez de vientos con orientación Oeste-Este durante el otoño anterior. Esta hipótesis, sin embargo, no pudo corroborarse, debido a la ausencia de registro meteorológico preciso para la zona estudiada.

Se corroboró la hipótesis de un efecto de la influencia de la dirección del viento sobre los patrones de distribución de los renovales. La prueba de comparación de pendientes mostró que la densidad de renovales de $P$. menziesii es mayor en las orientaciones favorecidas por la dirección del viento (ver modelos 3 y 4). Este patrón coincide con el encontrado por Sarasola et al. (2006), quienes analizaron la distribución

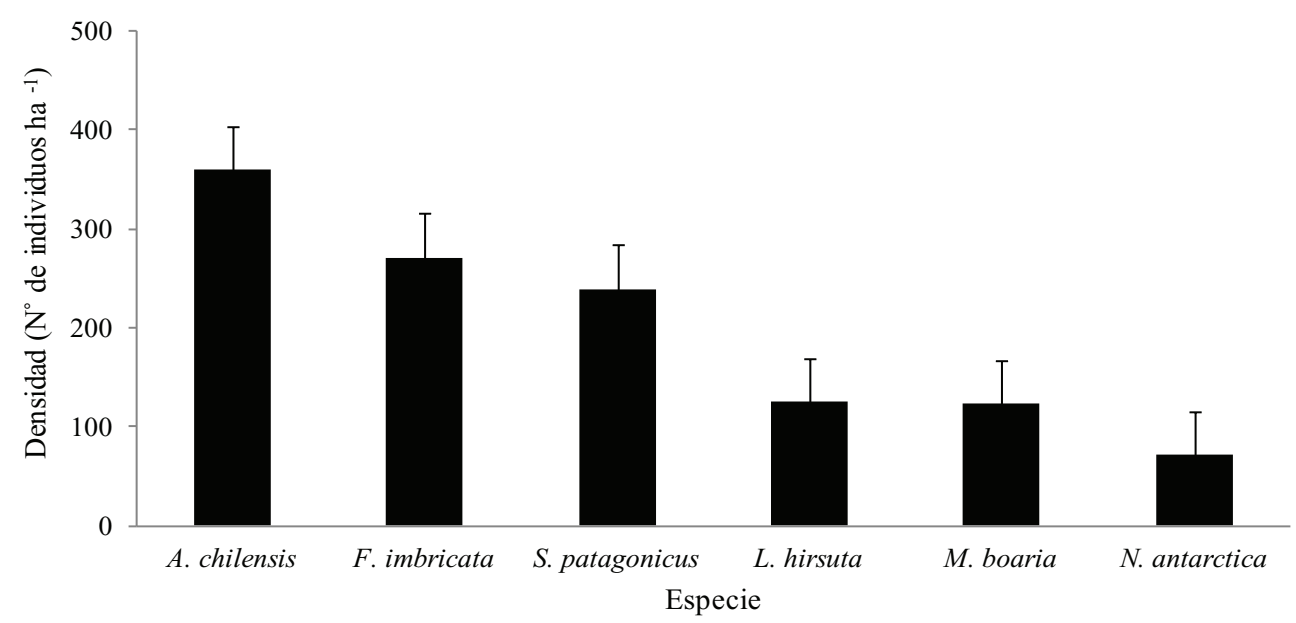

Figura 5. Abundancia de renovales de $P$. menziesii asociados a diferentes especies leñosas del sotobosque.

Pseudotsuga menziesii sapling abundance associated to different woody species of the understory. 
de renovales de $P$. menziesii ( $\mathrm{n}=6$ sitios) y Pinus contorta $(\mathrm{n}=8$ sitios) en Neuquén, en orientaciones favorecidas y no favorecidas y encontraron una fuerte influencia de la dirección del viento sobre la distribución de los renovales. Sin embargo, en otro trabajo realizado en Isla Victoria, Simberloff et al. (2002) analizaron el patrón de distribución de renovales en una plantación ( $\mathrm{n}=1$ sitio), y no encontraron influencia de la dirección del viento en el mismo.

Se encontró una mayor densidad de renovales de $P$. menziesii asociada a especies leñosas que a suelo desnudo o herbáceas: el sotobosque de los bosques de $A$. chilensis y de los matorrales mixtos estudiados, al no ser demasiado cerrados, proporcionan niveles de sombra suave, que facilitarían el establecimiento de la regeneración de P. menziesii. $\mathrm{Al}$ cabo de unos años y debido al rápido crecimiento de $P$. menziesii, en comparación con el crecimiento de las especies nativas de la zona, los renovales acceden a mayores niveles de luz, y logran sobrepasarlos en altura y establecerse de manera exitosa. Los renovales de P. menziesii se encontraron asociados a 22 especies leñosas y enredaderas. Hay seis especies de sotobosque a las que se los encontró asociados con mayor frecuencia: A. chilensis, F. imbricata, S. patagonicus, L. hirsuta, M. boaria y N. antarctica, no habiendo diferencias significativas en las densidades asociadas a cada una de las especies. Estos resultados demuestran que las especies leñosas, ejercen un efecto positivo sobre el establecimiento de los renovales de P. menziesii. Resultados similares encontraron Tejera y Davel (2004) quienes observaron que $S$. patagonicus, L. hirsuta y M. boaria actúan como facilitadoras para el establecimiento de plantines de $P$. menziesii llevados a campo. Por otro lado, Raffaele y Veblen (1998) y Rovere (2000), encontraron que A. chilensis, también se establece en asociación con S. patagonicus y L. hirsuta. Estas especies, tan frecuentes en los bosques de $A$. chilensis o en los matorrales mixtos actuarían como facilitadoras para el establecimiento de especies arbóreas.

En algunos trabajos se ha observado que el establecimiento de plántulas ha sido afectado negativamente por la presencia de herbáceas, las cuales compiten por agua y nutrientes (Hermann y Lavender 1990, Bava y Rechene 2004), por lo que en futuras investigaciones sería importante analizar por separado la densidad de renovales de P. menziesii asociados a herbáceas o a suelo desnudo. En bosques de $A$. chilensis se ha observado que la cobertura de herbáceas facilita la emergencia de especies juveniles arbóreas por el efecto combinado de amortiguar los extremos de humedad, proveer mejores microambientes que el suelo desnudo u hojarasca para la germinación y reducir la susceptibilidad a la depredación de semillas (Rovere 2000). También existen registros en los cuales se ha corroborado que la germinación y el crecimiento de distintas especies de coníferas exóticas son mayores sobre suelo desnudo (Cattaneo 2002). Peña y Pauchard (2001) observaron que P. menziesii se establece mejor en suelos desnudos o con escasa cobertura vegetal. Los resultados de este trabajo sugieren que las semillas de $P$. menziesii son capaces de ger- minar en diferentes tipos de condiciones de microambiente (e.g. suelo desnudo, herbáceas, o asociados a diferentes arbustos nativos), pero el establecimiento se ve favorecido por la presencia de especies leñosas del sotobosque.

Para prevenir procesos de invasión en áreas naturales vecinas a las forestaciones, es necesario implementar tareas de control. Las mismas deberían poner el énfasis en el control de la regeneración en edad reproductiva (presencia de conos), principalmente en las orientaciones favorecidas por la dirección del viento. Otra medida que permitiría minimizar la dispersión de los propágulos hacia las comunidades nativas, es instalar cortinas de especies arbóreas, en las áreas de borde de las plantaciones que lindan con bosque nativo, especialmente si las plantaciones se encuentran orientadas al Oeste de los bosques. Para Chubut, Río Negro y Neuquén, se propone utilizar cortinas de Populus nigra L. (álamo negro), que podrían actuar como una barrera mecánica, que minimice la dispersión de las semillas hacia los sectores aledaños. La especie, se usa desde hace más de un siglo en Patagonia y no produce invasiones. Por otro lado, alcanza alturas similares o superiores a las de los pinos, cuando estos están próximos a alcanzar su turno de corta. Otra ventaja que presenta el álamo negro, es que presenta altos contenidos de humedad en combustible vivo (Bianchi y Defossé 2015), lo cual le confiere una baja inflamabilidad y contribuiría a minimizar los riesgos de incendio.

Finalmente cabe mencionar, que a partir de la aplicación de la Ley Nacional No 26.331 "Presupuestos Mínimos de Protección Ambiental de los Bosques Nativos", se reguló el uso de los bosques nativos. En este marco, las provincias realizaron la ordenación de sus territorios boscosos, asignándoles una de tres categorías de conservación: Categoría I (rojo), sectores de alto valor de conservación que no deben transformarse, Categoría II (amarillo), sectores de mediano valor de conservación y Categoría III (verde), sectores de bajo valor de conservación que pueden transformarse parcialmente o en su totalidad. En las áreas con Categorías I y II no se puede realizar nuevas forestaciones y desmontes, sin embargo, persisten forestaciones realizadas con anterioridad a la aplicación de la Ley $\mathrm{N}^{\circ} 26.331$, en las cuales el manejo de la regeneración de $P$. menziesii o de otras especies exóticas, sigue sin realizarse. Así mismo, hay áreas de bosque nativo dentro de la Categoría III, que aún son susceptibles de ser forestadas. En estos casos, se sugiere que las plantaciones de $P$. menziesii sean instaladas a más de 120 metros de bosque nativo, y acompañadas de planes de manejo de la regeneración.

\section{AGRADECIMIENTOS}

A los revisores por sus valiosos aportes, los mismos permitieron mejorar sustancialmente el manuscrito. A Mariano Gómez $(\dagger)$ por su calidad humana y su asesoramiento en SIG. A Adriana Kutschker y Pablo López Bernal por sus aportes en los análisis estadísticos. Al guardaparque, Danilo Hernández Otaño, del Parque Nacional Los Alerces por su 
amabilidad y disponibilidad de tiempo. A quienes nos asistieron en la toma de datos a campo: César M. Acheritobehere, Jorge Beatove, Sofía López, Valeria Silva y Luis Epele.

\section{REFERENCIAS}

Bava J, C Rechene. 2004. Dinámica de la regeneración de lenga (N. Pumilio (Poepp. et Endl. Krasser)) como base para la aplicación de sistemas silvícolas. In Arturi M, J Frangi, J Goya eds. Ecología y manejo de los bosques de Argentina. La Plata, Argentina. Laboratorio de Investigaciones de Sistemas Ecológicos y Ambientales. Facultad de Cs. Agr. y Forestales-Fac. de Cs. Nat. y Museo, Universidad Nacional de La Plata. p. 1-24.

Bianchi LO, GE Defossé. 2015. Live fuel moisture content and leaf ignition of forerts species in Andean Patagonia, Argentina. International Journal of Wildland Fire 24(3): 340-348.

Cattaneo M. 2002. Effects of microsite characteristics, competition and grazing on Pinus contorta Dougl. and Pseudotsuga menziesii (Mirb.) Franco seedling establishment. Tesis Master de Ciencias Forestales. Christchurch, New Zealand. Universidad de Canterbury. 153 p.

Chalcoff VR, CL Morales, MA Aizen, Y Sasal, AE Rovere, M Sabatino, C Quinteros, M Tadey. Interacciones plantas animal, la polinización. 2014. In Raffaele E, M de Torrres Curth, CL Morales, T Kitzberger eds. Ecología e Historia Natural de la Patagonia Andina. Un cuarto de siglo de investigación en biogeografía, ecología y conservación. Buenos Aires, Argentina. Editorial Félix de Azara. p. 113-132.

Davel M. 2008. Estimación de la productividad de sitio. In Davel $\mathrm{M}$ ed. Establecimiento y manejo del pino oregón en Patagonia Esquel, Argentina. Esquel, Argentina. CIEFAP. p. 30-42.

Di Rienzo JA, F Casanoves, MG Balzarini, L González, M Tablada, CW Robledo. 2008. InfoStat, versión 2008, Grupo InfoStat, FCA. Córdoba, Argentina. Universidad Nacional de Córdoba.

Gobbi M, T Schlichter. 1998. Survival of Austrocedrus chilensis seedlings in relation to microsite conditions and forest thinning. Forestal Ecology and Management 111(2): 137-146.

Hermann RK, DP Lavender. 1990. Pseudotsuga menziesii (Mirb.) Franco. In Burns RM, BH Honkala eds. Silvics of North America Volume 1 Conifers. Washington, USA. Forest Service. p. 527-540. (Agriculture Handbook 654)

IUCN (Unión Internacional para la Conservación de la Naturaleza, CH). Species Survival Commission, and Natural Resources. Species Survival Commission. IUCN Red List Categories and Criteria. Consultado 10 abr. 2014. Disponible en http://www.iucnredlist.org.

Kitzberger T, DF Steinaker, TT Veblen. 2000. Effects of climatic variability on facilitation of tree establishment in Northern Patagonia. Ecology 81(7): 1914-1924.

Letourneau FJ, E Andenmatten, T Schlichter. 2004. Effect of climatic conditions and tree size on Austrocedrus chilensis - shrub interactions in northern Patagonia. Forest Ecology and Management 191(1): 29-38.
Martínez M. 1982. Vientos. In Centro Editor de América Latina eds. Atlas Físico de la República Argentina Volumen 2. Buenos Aires, Argentina. p. 216-221.

Martyniuk NA, CL Morales, MA Aizen. 2014. Invasive conifers reduce seed set of a native Andean cedar through heterospecific pollination competition. Biological Invasions 17(4): 1055-1067.

Nuñez CI, E Raffaele, MA Nuñez, F Cuassolo. 2009 a. When do nurse plants stop nursing? Temporal changes in water stress levels in Austrocedrus chilensis growing within and outside shrubs. Journal of Vegetation Science 20(6): 1064 - 1071.

Nuñez MA, TR Horton, D Simberloff. 2009 b. Lack of belowground mutualisms hinders Pinaceae invasions. Ecology 90(9): 2352-2359.

Orellana IA, E Raffaele. 2010. The spread of the exotic conifer Pseudotsuga menziesii in Austrocedrus chilensis forests and shrublands in northwestern Patagonia, Argentina. New Zealand Journal of Forestry Science 40: 199-209.

Pauchard A, MA Nuñez, E Raffaele, RO Bustamante, N Ledgard, MA Relva, D Simberloff. 2010. Symposium summary: Introduced conifer invasions in South America: an update. Frontiers of Biogeography 2(2): 34-36.

Peña E, A Pauchard. 2001. Coníferas introducidas en unidades del SNASPE: un riesgo para la biodiversidad. Bosque Nativo 30(1): 3-7.

Raffaele E, TT Veblen. 1998. Facilitation by nurse shrubs of resprouting behavior in a post-fireshrubland in northern Patagonia, Argentina. Journal of Vegetation Science 9(5): 693-698.

Relva MA, MA Nuñez. 2014. Factores que facilitan y retrasan la invasión de coníferas exóticas en la Isla Victoria. Ecología Austral 24(2): 145-153.

Richardson DM, BW van Wilgen, MA Nuñez. 2007. Alien conifer invasions in South America: short fuse burning? Biological Invasion 10(4):573-577.

Rovere AE, AC Premoli. 2005. Dispersión asimétrica de semillas de Embothrium coccineum (Proteaceae) en el bosque templado de Chiloé, Chile. Ecología Austral 15(1): 1-7.

Rovere AE. 2000. Condiciones ambientales de la regeneración del ciprés de la cordillera (Austrocedrus chilensis). Bosque 21(1): 57-64.

Salgado Salomón ME, C Barroetaveña, M Rajchenberg. 2013. Pseudotsuga menziesii invasion in native forests of Patagonia, Argentina: What about mycorrhizas? Acta Oecologica 49: 5-11.

Sarasola MM, VE Rusch, TM Schilichter, CM Ghersa. 2006. Invasión de coníferas forestales en áreas de estepa y bosque de ciprés de la cordillera en la Región Andino Patagónica. Ecología Austral 16(2): 143-156.

Simberloff D, M Relva, M Nuñez. 2002. Gringos en el bosque: introduced tree invasion in a native Nothofagus/Austrocedrus forest. Biological Invasions 4(1-2): 35-53.

Tejera L, MM Davel. 2004. Establecimiento de pino oregón en Patagonia. Patagonia Forestal 10(3): 9-12.

Williams CG, SL LaDeau, R Oren, GG Katul. 2006. Modeling seed dispersal distances: Implications for transgenic Pinus taeda. Ecological Applications 16(1): 117-124. 
